ISSN: 2637-4722

\title{
Newborn With 21 Trysomy and Chilous pleural Effusion
}

\author{
Khatuna Lomauri* \\ *Department of Neonatal Intensive Care Unit, Tbilisi State Medical University, Georgia, UK
}

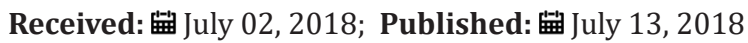

*Corresponding author: Khatuna Lomauri, Department of Neonatal Intensive Care Unit, Tbilisi State Medical University, Georgia, UK

\begin{abstract}
Neonatal chylothorax results from the accumulation of chyle in the pleural space and may be either congenital or an acquired condition. Congenital chylothorax is most likely due to abnormal development or obstruction of the lymphatic system and often associated with hydropsfetalis. It can be idiopathic or may be associated with various chromosomal and other genetic abnormalities. It is important to identify infants with chylothorax, as there are specific issues that need to be addressed in the management of these patients. In the neonate, chylous effusion is a common cause of pleural effusions and characterized as an exudate because of the high protein and lipid content once the infant is fed. The fluid will be clear/yellow to slightly cloudy in the unfed state and will quickly become milky following feeding, as chylomicrons appear in the fluid. Lymphocytes predominate in the differential cell count of chyle. The volume of fluid output can be high, and management can be challenging.

We present a case of newborn with 21 trisomy who developed moderate RDS, chest X-ray and US reveal pleural effusion on right side, rapid intervention was made before deterioration, requiring intensive life-saving measures. We review the common manifestations of congenital chylotoraxes and emphasize the importance of early diagnosis and intervention in preventing devastating outcomes from this condition.
\end{abstract}

Keywords: Chylothorax; Pleural Effusion; Respiratory Distress Syndrome (RDS); Positive Pressure Ventilation (PPV)

\section{Case Presentation}

\section{Prenatal and Birth Histories}

Mom was a 35yo G2P2 who came into L\&D at 37+5/7wks in active labor. She did not receive antenatal steroids or antibiotics. Serology: A+, RPR neg, Hep B neg, HIV neg, GBS status - unknown. It was spontaneous vaginal delivery of a male infant with trisomy 21 , which was diagnosed antenatally. The pediatric team was called to a "code blue" for fetusbradycardia. At delivery, the infant had a $\mathrm{HR}>100 / \mathrm{min}$, but no respiratory effort, and was limp and blue. He required PPV (\& Oxygen) with Ambu Bag and color/tone improved. Apgar Scores were 2 and 7 at 1 and 5minutes, respectively. BW was 2800 grams.

\section{Physical Examination at Birth}

a) Head: Flat occiput; up-slanting palpebral fissures; bilateral epicanthal folds; flat nasal bridge; hypertelorism; b) Oral Cavity: Moist pink mucosae; intact palate; normal sucking and rooting reflex;

c) Lungs: Moderate respiratory distress, auscultation breath sounds not equal bilaterally; especially less on the right side;

d) Cardiovascular: First and second heart sounds heard; regular rate and rhythm; grade 3/6 murmur noted over left sternal border;

e) Abdomen: Not distended; soft; no organomegaly; not tender; umbilicus clean with 3 vessels;

f) Genitourinary: Normal male genitalia, patent anus;

g) Skeletal: Normal Semian line on hands, wide gap between 1-st and 2-nd toes on feet;

h) Skin: no rash or birthmarks. 


\section{Clinical Condition}

\section{Within First 2hrs}

Respiratory status: dyspnea, SpO2 -92\%, with FiO2 45\%; Echo: mild to moderate VSD and PDA; X-ray and Chest Ultrasound: pleural effusion on right side; Abdominal US: with no pathology; Normal micturition and defecation. Lab studies: CBC - lymphocitopenia, CRP - 16mg/dl; Capillary blood gas: 7.25/65/28/+1 - respiratory acidosis; Thoracocentesis was performed, with approximately $60 \mathrm{~mL}$ fluid obtained for culture and diagnostic studies; blood culture was obtained, amp/gen was given;

\section{After 8hrs}

Respiratory status remains unchanged: tachypnea, mild sternal retraction. X-ray: with no improvement; Chest US: effusion on right pleural space, covering almost all right lung; Chest tube was inserted; CT scan: no malignancy in mediastinum;

\section{Pleural Fluid Analysis}

\section{On Fasting}

Clear slight cloudy; pH: 7.45; White blood cell count: $3000 / \mu \mathrm{L}\left(3.00 \times 10^{9} / \mathrm{L}\right)$; Red blood cell count: $39 / \mu \mathrm{L}(0.039 \times$ 109/L); Lymphocytes: 90\% (0.90); Triglycerides: 1,120mg/dL (12,5mmol/L); Cholesterol: $70 \mathrm{mg} / \mathrm{dL}(1.8 \mathrm{mmol} / \mathrm{L})$;Monocytes: 4\% (0.4); Glucose: 49mg/dL (2,7mmol/L); Protein: 2,8g/dL (28g/L); Albumin: 2.2g/dL (22g/l).

\section{After Giving LCT Formula}

Milky Yellow Turbid Fluid; pH: 7.52; White blood cell count: $3250 / \mu \mathrm{L}\left(3.25 \times 10^{9} / \mathrm{L}\right)$; Red blood cell count: 33/ $\mu \mathrm{L}(0.033 \times$ 109/L); Lymphocytes: 96\% (0.96); Triglycerides: $1,500 \mathrm{mg} / \mathrm{dL}$ (16.95mmol/L); Cholesterol: $72 \mathrm{mg} / \mathrm{dL}(1.9 \mathrm{mmol} / \mathrm{L}) ;$ Monocytes: 4\% (0.4); Glucose: $89 \mathrm{mg} / \mathrm{dL}$ (4.9mmol/L); Protein: $3.2 \mathrm{~g} / \mathrm{dL}$ (32g/L); Albumin: 2.6g/dL (26g/l);

\section{Management}

a) Pleural drainage and measument of chyle flow were done;

b) NPO and nutritional support with TPN; c) TPN was weaned off 5 days after the introduction of MCT formula; No breast Milk!

The infant was discharged home on day 36 with instructions to continue taking the medium chain triglyceride-based formula until re-evaluation follows up [1,2] (Figure 1).

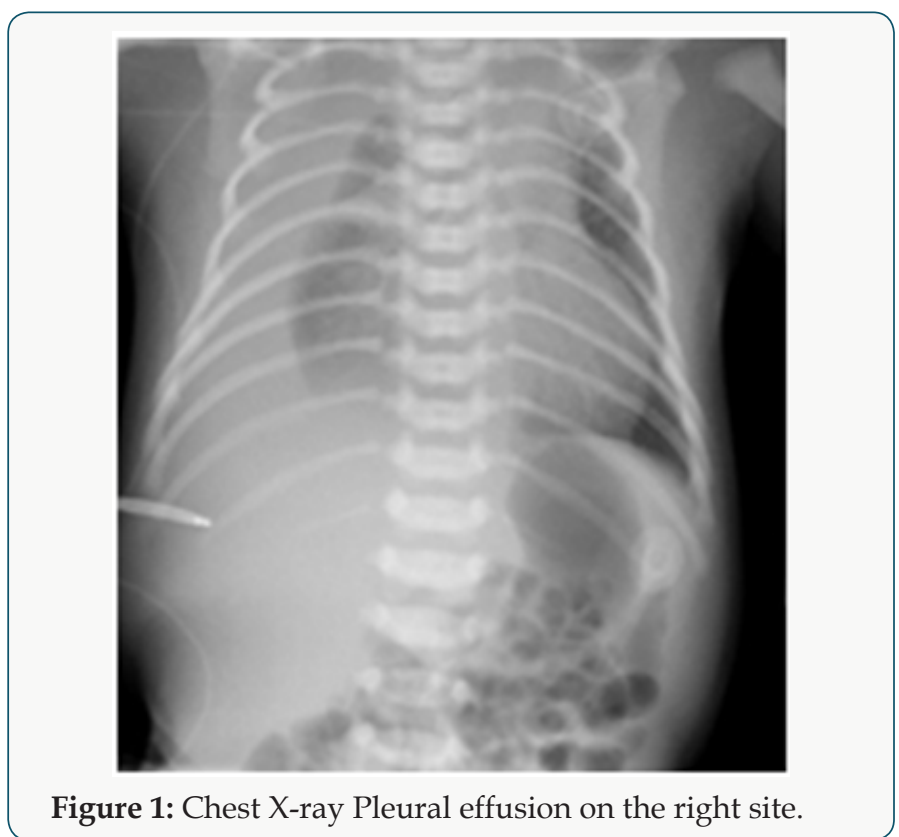

\section{Conclusion}

Congenital Chylothorax is a rare condition with multiple etiologies. Pleural fluid analysis can identify this condition when clinical suspicion exists. Conservative management is generally recommended in most cases with surgery being reserved for those that have a large or persistent leak or in those who become immunologically challenged or malnourished.

\section{References}

1. Rocha G, Fernandes P, Rocha P (2006) Pleural effusions in the neonate. Acta Paediatr 95(7): 791.

2. Van Straaten HL, Gerards LJ, Krediet TG (1993) Chylothorax in the neonatal period. Eur J Pediatr 152(1): 2-5.

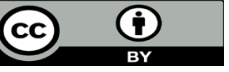

This work is licensed under Creative Commons Attribution 4.0 License

To Submit Your Article Click Here:

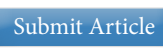

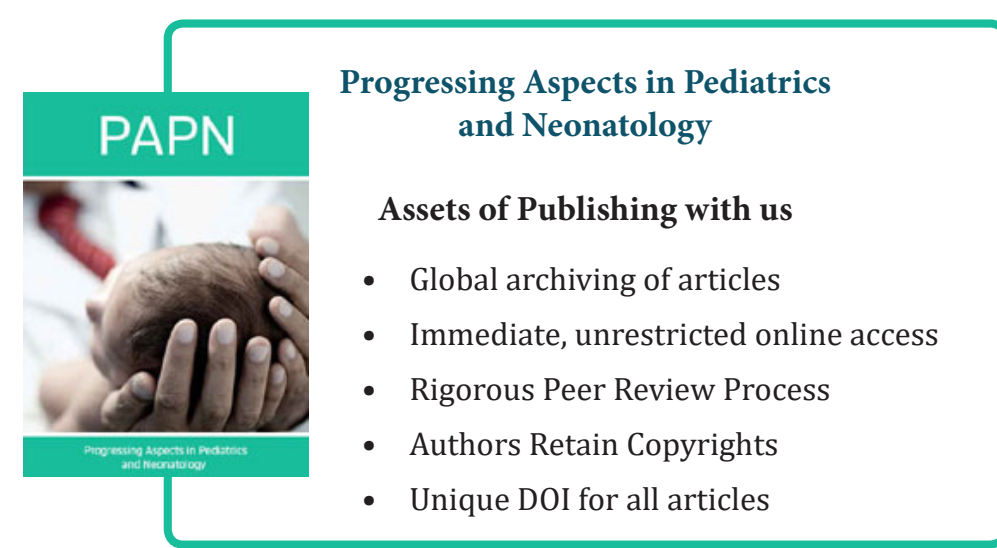

DOI: 10.32474 PAPN.2018.01.000120 